\title{
Deux disques de musique gnawa
}

\section{Luc Weissenberg}

\section{(2) OpenEdition \\ Journals}

Édition électronique

URL : http://journals.openedition.org/ethnomusicologie/219

ISSN : 2235-7688

Éditeur

ADEM - Ateliers d'ethnomusicologie

Édition imprimée

Date de publication : 1 décembre 2001

Pagination : 342-345

ISBN : 2-8257-07-61-9

ISSN : $1662-372 X$

Référence électronique

Luc Weissenberg, « Deux disques de musique gnawa », Cahiers d'ethnomusicologie [En ligne], 14 | 2001, mis en ligne le 10 janvier 2012, consulté le 19 avril 2019. URL : http://journals.openedition.org/ ethnomusicologie/219

Ce document a été généré automatiquement le 19 avril 2019.

Tous droits réservés 


\title{
Deux disques de musique gnawa
}

\author{
Luc Weissenberg
}

\section{RÉFÉRENCE}

Gnawa de Mostaganem. Rituels de la Layla et du Moussem. Enregistrements (1971), textes et photos réalisés par Henri Lecomte ; transcription et traduction des textes des chants : Abdelhafid Chlyeh . Notice bilingue français/anglais de 12 pages. 1 CD Iris Music 3001 839, 2000.

Gaâda. Diwane de Béchar. Enregistrements et mixages : François Brissot ; textes : Abdel Laoufi ; photos : Nora Zanoun, Abdou ; traduction anglaise : Farès Bouchia. Notice bilingue français/anglais de 14 pages, 10 photos noir \& blanc. 1 CD Samarkand SKD002, 1999.

1 Immanquablement, la sortie d'un nouvel opus de musique gnawa sur le marché du disque ne peut que rappeler l'engouement actuel suscité par cette musique, porteuse d'un certain " envoûtement ", d'une "transe ", d'un " groove festif », ce que reflète d'ailleurs très bien la maquette $d u$ premier $C D$, représentant la scène musicale dans une atmosphère havane baignant dans le halo diffus des encens... Ceci en fait un produit recherché sur le marché du disque, bien que l'Occident s'y soit intéressé dès les années 1950 à travers Paul Bowles, et plus tard par les expériences parfois très réussies de " fusion » avec le jazz (Randy Weston, Pharoah Sanders, Don Cherry, Steve Lacy, Dizzy Gillespie, Graham Haynes...), avec le rock (Led Zepplin), la musique folk (Nass El Ghiwan), la world music (Hassan Hakmoun, Pojma) ou le ragga (Gnawa Diffusion, Orchestre National de Barbès), pour n'en citer que quelques-uns. Enfin, certaines collections de disques de musique «ethnique » semblent intéressées à posséder au moins un titre de musique gnawa "traditionnelle», enregistrée de préférence in situ (catalogues Al Sur, Ocora, etc.).

2 Le présent $\mathrm{CD}$ s'inscrit dans cette dernière catégorie en présentant la musique de la confrérie Saiddiya de Mostaganem (dans le Nord-Ouest algérien). Les Gnawa appartiennent en effet à une confrérie populaire - une forme de soufisme maghrébin - 
issue du contexte religieux et culturel du maraboutisme en Afrique du Nord. Comme les autres confréries présentes au Maghreb (et surtout au Maroc: 'Ayssawa, Hamadsha, Jilala, etc.), les Gnawa accordent une place centrale dans leurs rituels extatiques à la musique, pour appeler les génies et accompagner les états de possession. Leur chaîne initiatique les relie à un personnage important de l'islam, le premier muezzin et esclave noir affranchi devenu proche compagnon du Prophète : Sidi Bîlâl, ce qui leur vaut le nom de Bilali en Algérie. Témoin de leur histoire mouvementée, le riche répertoire choreutico-musical gnawa intègre des éléments des cultures musicales arabe, juive, berbère, andalouse, en plus d'apports de l'Afrique Noire (Bambara, Songhaï, Sokole, Haoussa, Foulbé).

Comme l'indique le sous-titre, les extraits presentés ici sont donc issus de deux rituels importants: la lila (orthographié ici layla) tout d'abord, qui désigne la célébration nocturne durant laquelle se pratique le rite de possession (derdeba), se déroulant chaque vendredi à Mostaganem (au moment de l'enregistrement en 1971) dans une maison appartenant à la confrérie. Puis le moussem, une célébration annuelle autour du sanctuaire de Sidi Mohammed Mejdoûb. On suppose, à défaut d'indications claires, que les plages 1 à 3 se rapportent à la première, et les plages 4 à 6 à la seconde.

Dans la première partie, l'auditeur se fera une idée de l'architecture rituelle de la lila, dans laquelle s'ordonne une série de pièces indépendantes les unes des autres, qui sont des invocations vocales et des hymnes (où l'on chante la grandeur et la gloire de Dieu et du Prophète Mohammed), des incantations aux saints protecteurs (qu'on évoque par leur nom) et des mélodies particulières aux génies mluk (chaque melk a sa devise musicale, une courte phrase qui lui est propre - par exemple Sidi Hammou invoqué dans la plage 1, qui est un melk « rouge ») conduite par le m'allem, joueur de l'instrument central guembri, un luth-tambour à trois cordes de longueurs inégales (une haute, une médiane et une basse) à registre grave. De plus, à la voix basse des cordes en boyau du guembri - amplifiée par une caisse de résonance en bois et une table harmonique en peau - répond le tintement du sistre métallique (sersera) inséré sur le bout du manche.

Autour de lui, un noyau de musiciens-danseurs initiés (peut-être une dizaine) jouent des crotales métalliques qaraqeb et assurent le chœur, auquel se joint le reste des participants, également familiers des chants et des rythmes. Les crotales assurent un soubassement rythmique régulier pour chaque phase, alors que le chant est fluctuant. On est ainsi immédiatement plongé dans le caractère violent, voir assourdissant (derdeba signifie littéralement "grand bruit») d'une polyrythmie complexe (changements brusques, accélérations soudaines...). Ces prises ont été effectuées après les morceaux préliminaires - qui nécessitent les tambours tbal -, leur auteur ayant reçu la consigne de ne commencer l'enregistrement « que lorsque les pièces de monnaie offertes par les fidèles tenaient sur le front du maâlem »! Aussi l'intensité du volume sonore des instruments de percussions dans la lila est bien rendu par l'enregistrement, et l'auditeur est littéralement écrasé par le martèlement en staccato des qaraqeb (qui donnent la clé du code rythmique: des combinaisons complexes permettant le balancement et l'interpénétration entre le ternaire et le binaire). Cela contraste nettement avec la faible intensité sonore du guembri, et l'auditeur habitué aux enregistrements digitaux devra se contenter de l'exposition des thèmes en introduction (plages 1 et 2). Il en est de même pour les paroles, totalement inaudibles la plupart du temps (plage 3), ce qui achèvera de convaincre l'auditeur de l'authenticité de cet enregistrement en " prise directe »...

6 La seconde partie, enregistrée en août 1971 lors des processions rituelles (aâda) dans les jours qui précèdent le sacrifice annuel du taureau (dbiha) par la confrérie, suit 
« l'orchestre du stâmbâli constitué par des joueurs de crotales métalliques, les qarqabou, et de tambours cylindriques, les ganga». On ne sait pas vraiment quels moments de la séquence rituelle sont présentés sur le $\mathrm{CD}$ (procession vers la mer, remontée vers le sanctuaire ?). On n'en apprend guère plus sur les ganga, bien que le timbre et la technique le jeu ressemblent fort à ceux du grand tambour tbal à deux membranes, frappées par deux bâtons en bois. L'exécution dynamique des percussions, avec des variations d'accents et de divisions temporelles, est particulièrement saisissante sur la plage 5, quoiqu'on prenne la séquence en cours de route, et qu'ici encore, l'enregistrement ne permette pas une réelle écoute.

7 On regrettera donc à la fois la faible qualité sonore des enregistrements : confusion générale, brusque chute du volume à la plage 1 (4'40") et surgissement de sons incongrus à la plage 2 (1'16 et 1'26"), et surtout manque d'une quelconque justification dans la notice, qui se contente de descriptions générales. En effet, on ne peut s'empêcher de se demander pour quelle raison on édite aujourd'hui de vieux enregistrements d'une telle qualité sonore. La confrérie aurait-elle cessé ses activités, ces enregistrements constituant alors un document d'archive? Si tel est le cas, pourquoi omettre les informations sur le moment précis des enregistrements dans la séquence rituelle (au moins pour distinguer clairement la lila du moussem), voire les noms des musiciens. Une transcription des différents rythmes et une translittération plus précise des chants n'aurait certainement pas été inutile. Enfin, on regrettera que « Allah » soit orthographié «Hallah» au dos du CD, ce dernier détail étant peut-être paradigmatique d'une production aussi approximative qu'expéditive, ce qui ne rend certainement pas justice à la complexité et la qualité esthétique de ces pièces.

8 La démarche du groupe Gaâda est toute différente, et la simple juxtaposition de ce CD et du précédent signale déjà l'hétérogénéité de la production musicale autour de la musique gnawa. Formé autour de la communauté becharienne (Sud de l'Algérie) de Paris, il s'agit d'abord d'un " groupement culturel » dans les années 70, qui réunit dans un but festif "passer du temps et évoquer les souvenirs de jeunesse au pays" - des travailleursmusiciens, bientôt rejoints par des compatriotes venant d'autres régions, pour finalement s'orienter vers le style musical du diwane, un genre musical que les auteurs décrivent comme mystique et populaire, et pratiqué par des artisans et des ouvriers. Le diwane, terme qui signifie "réunion des adeptes d'une confrérie mystique ", correspond donc à un cérémonial à l'origine pratiqué par les descendants d'esclaves (le «Diwane de Sidi Bilal » en Algérie), et qui s'est popularisé. « Diwane » prend également pour les auteurs le sens d'une assemblée, une rencontre, et une "fraternisation entre les peuples noirs, arabes, et berbères ». Ainsi, des cultes extatiques du Diwane original, le groupe Gaâda, en " voyage " à Paris, en banlieue ou en province, veut perpétuer "l'état d'esprit », en adaptant et en enrichissant le répertoire au gré des rencontres intercomunautaires et intergénérationelles. On perçoit bien, au-delà du projet artistique, un discours philosophique et politique, qui se traduit dans leur musique, simple et chaleureuse.

Il s'agit de morceaux traditionnels, adaptés ou composés par ce sextet, calqués sur la structure musicale gnawa (présentation du motif au guembri, chant et cœur responsoriel, apports rythmiques). Ils sont exécutés avec soin, de même que les arrangements, dont l'aspect parfois peu « traditionnel » (effets de réverbération) contribue à mettre en valeur l'instrumentation. De plus, l'intégration dans l'orchestre d'instruments à cordes tels la mandole et le banjo (ce qui n'est pas sans faire penser au groupe de pop gnawa des années 70 Nass El Ghiwane), et des percussions (bendir, tarija, derbouka), apporte des timbres 
originaux et rend leur musique particulièrement dynamique, tout en masquant l'aspect assourdissant des crotales et des tambours. Suivent les belles prestations vocales des six musiciens, en solo (notamment plage 2 et 4 ) ou en chœur. Au gré des morceaux, les thèmes chers au groupe défilent : le « voyage » que peut déclencher l'odeur du thé vert et de l'encens (plage 1), l'harmonie suscitée par les chants de louange au saint (plages 2 et 4), le dépassement de soi dans l'évocation divine (plage 3), l'hommage à Sidna Bilal (plage 5), et l'anneau comme symbole d'unité (plage 6), autant de chants qui exaltent l'idée d'une fraternité mystique.

On a donc affaire avec ce $\mathrm{CD}$ à un bel exemple d'adaptation du répertoire de musique gnawa « désacralisée » et librement arrangée à des fins purement esthétiques, et non plus rituelles. Si la musique de Gaâda ne peut être identifiée à une musique "traditionnelle ", elle n'en est pas moins " authentique ", tout comme la notice du CD qui oblige l'auditeur qu'il aime ou pas - à reconnaître l'honnêteté de la démarche du groupe qui tente, en jetant un pont au-dessus de la Méditerranée, de «concilier tradition et modernité, et favoriser le terrain de la compréhension mutuelle ». On ne peut que les en remercier.

\section{BIBLIOGRAPHIE}

CHLYED Abdelhafid, dir., 1999, L'univers des gnaoua. Casablanca : La Pensée Sauvage/ Le Fennec.

HELL Bertrand, 2000, Possession et Chamanisme. Les maîtres du désordre. Paris : Flammarion. 